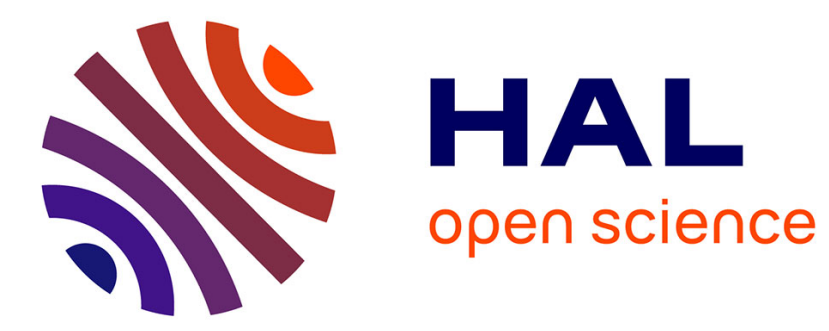

\title{
Stable Poiseuille flow transfer for a Navier-Stokes system
} Rafael Vazquez, Emmanuel Trélat, Jean-Michel Coron

\section{To cite this version:}

Rafael Vazquez, Emmanuel Trélat, Jean-Michel Coron. Stable Poiseuille flow transfer for a NavierStokes system. 2006, 6 p. hal-00086477

\section{HAL Id: hal-00086477 https://hal.science/hal-00086477}

Submitted on 18 Jul 2006

HAL is a multi-disciplinary open access archive for the deposit and dissemination of scientific research documents, whether they are published or not. The documents may come from teaching and research institutions in France or abroad, or from public or private research centers.
L'archive ouverte pluridisciplinaire HAL, est destinée au dépôt et à la diffusion de documents scientifiques de niveau recherche, publiés ou non, émanant des établissements d'enseignement et de recherche français ou étrangers, des laboratoires publics ou privés. 


\title{
Stable Poiseuille Flow Transfer for a Navier-Stokes System
}

\author{
Rafael Vázquez, Emmanuel Trélat and Jean-Michel Coron
}

\begin{abstract}
We consider the problem of generating and tracking a trajectory between two arbitrary parabolic profiles of a periodic 2D channel flow, which is linearly unstable for high Reynolds numbers. Also known as the Poisseuille flow, this problem is frequently cited as a paradigm for transition to turbulence. Our approach consists in generating an exact trajectory of the nonlinear system that approaches exponentially the objective profile. A boundary control law guarantees then that the error between the state and the trajectory decays exponentially in the $L^{2}$ norm. The result is first proved for the linearized Stokes equations, then shown to hold for the nonlinear Navier-Stokes system.
\end{abstract}

\section{INTRODUCTION}

One of the few situations in which analytic expressions for solutions of the stationary flow field are available is the channel flow problem. Also known as the Poiseuille flow, this problem is frequently cited as a paradigm for transition to turbulence. Poiseuille flow requires an imposed external pressure gradient for being created and sustained [3]. The magnitude of the pressure gradient determines the value of the centerline velocity, which parameterizes the whole flow.

It is very well known that this solution goes linearly unstable for Reynolds numbers greater than the so-called critical Reynolds number, $R e_{C R} \approx 5772$ [11]. The problem of locally stabilizing the equilibrium has been solved by means of optimal control [8], and backstepping [17]. Observers have been developed using dual methods [18].

However, all prior works consider a constant pressure gradient and a developed flow which is already close to the desired solution. The problem of tracking time varying profiles generated by unsteady pressure gradients has, so far, not been considered from a control point of view. Stability for channel flow driven by unsteady pressure gradient has been previously studied [9].

In this paper we consider the problem of moving the state from one Poiseuille equilibrium to another. For example, we may wish to smoothly accelerate fluid at rest up to a given Reynolds number, probably over the critical value, avoiding transition to turbulence. The means at our disposal are the imposed pressure gradient and boundary control of the velocity field (only at one wall).

This is a problem of practical interest which, to the best of our knowledge, has not been solved or even been considered

R. Vázquez is with the Department of Mechanical and Aerospace Engineering, University of California San Diego, La Jolla, CA 92093-0411.

E. Trélat is with the Univ. Paris-Sud, Lab. Math., UMR 8628, 91405 Orsay Cedex, France.

Jean-Michel Coron is with the Institut Universitaire de France and Univ. Paris-Sud, Lab. Math., UMR 8628, 91405 Orsay Cedex, France.

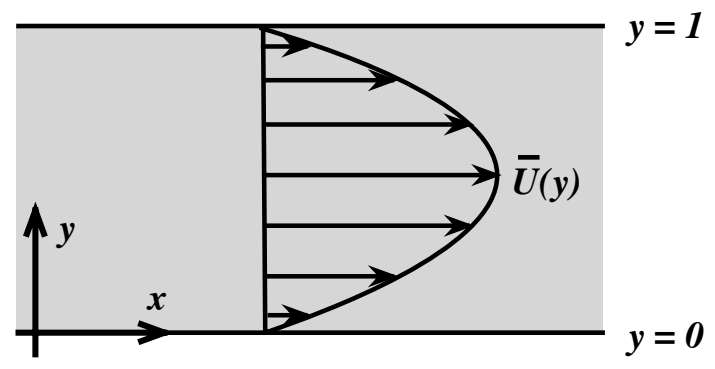

Fig. 1. 2D Channel Flow with an equilibrium profile

so far, since all control laws in the literature are designed for one given Poiseuille flow (fixed Reynolds number).

A possible solution for the problem would be to apply quasi-static deformation theory; this would require to modify the pressure gradient very slowly, and simultaneously gain-schedule a fixed Reynold number boundary controller like [17] for tracking a (slowly) time varying trajectory, which in general would not be an exact solution of the system. This idea has been already used for moving between equilibria of a nonlinear parabolic equation [4], or a wave equation [5]. Other applications include the shallow water problem [6] and the Couette-Taylor flow [12]. In this paper, however, we follow an alternative approach, finding an exact, fast trajectory of the system which is then stabilized by means of boundary control. The advantage of this approach is that it reaches the objective profile much faster.

The organization of the paper is as follows. We begin stating the model in Section II. In Section III, we solve the problem of generating an exact trajectory between two Poiseuille profiles. Section IV presents the boundary control laws and our main results. We follow with Section V, where we present the tools we use to solve the problem. Section VI presents a sketch of proof of the main results.

\section{Channel Flow MOdel}

We consider a 2-D incompressible fluid filling a region $\Omega$ between two infinite planes separed from each other a distance $L$, as shown in Fig. 1. Define $U_{c}$ as the maximum centerline velocity, $\rho$ and $\nu$ as the density and the kinematic viscosity of the fluid, respectively, and the Reynolds number, $R e$, as $R e=U_{c} h / \nu$. Then, using $L, L / U_{c}$ and $\rho \nu U_{c} / L$ as length, time and pressure scales respectively, we can write 
the nondimensional 2-D Navier-Stokes equations as follows,

$$
\begin{aligned}
u_{t} & =\frac{\triangle u}{R e}-p_{x}-u u_{x}-v u_{y} \\
v_{t} & =\frac{\triangle v}{R e}-p_{y}-u v_{x}-v v_{y}
\end{aligned}
$$

where $u$ is the streamwise velocity, $v$ the wall-normal velocity, and $p$ the pressure, with boundary conditions

$$
\begin{aligned}
& u(t, x, 0)=v(t, x, 0)=0, \\
& u(t, x, 1)=U(t, x), \\
& v(t, x, 1)=V(t, x) .
\end{aligned}
$$

In (4) and (5), $U$ and $V$ are the actuators located at the upper wall. Additionally we consider an incompressible fluid, so the velocity field must verify in $\Omega$ the divergence-free condition

$$
u_{x}+v_{y}=0
$$

In this nondimensional coordinates, $\Omega$ can be defined as

$$
\Omega=\left\{(x, y) \in \mathbb{R}^{2}: 0 \leq y \leq 1\right\},
$$

with boundary $\partial \Omega=\partial \Omega_{0} \cup \partial \Omega_{1}$, where $\partial \Omega_{0}$ and $\partial \Omega_{1}$ are the lower and upper wall, respectively, and will be referred to as the uncontrolled and controlled boundary.

\section{TRAJECTORY GENERATION AND CONTROL OBJECTIVE}

The stationary family of solutions of (1)-(5) is the Poiseuille family of parabolic profiles, $\mathcal{P}^{\delta}$, which is described by a single parameter $\delta$ (the maximum centerline velocity) in the following way

$$
\mathcal{P}^{\delta}=\left(u^{\delta}, v^{\delta}, p^{\delta}\right)=\left(4 \delta y(1-y), 0,-\frac{8 \delta}{R e} x\right) .
$$

Velocity actuation at the wall is zero for $\mathcal{P}^{\delta}$, since both $u^{\delta}$ and $v^{\delta}$ are zero at the boundaries. The pressure gradient $p_{x}^{\delta}=-\frac{8 \delta}{R e}$ must be externally sustained [3].

Our first task is, given $\delta_{0}$ and $\delta_{1}$, generate an unsteady trajectory path $\Theta(t)=(u(t), v(t), p(t))$, where space dependence is omitted for clarity, connecting $\mathcal{P}^{\delta_{0}}$ to $\mathcal{P}^{\delta_{1}}$. We assume $\delta_{0}=0$ and $\delta_{1}=1$ for simplicity.

Consider the trajectory $\Theta^{q}(t)$ defined as

$$
\Theta^{q}(t)=\left(u^{q}(t), v^{q}(t), p^{q}(t)\right)=(g(t, y), 0, x q(t)),
$$

where $q$ is the chosen external pressure gradient. Then, by substitution we see that (9) verifies (1)-(5) whenever

$$
g_{t}=\frac{g_{y y}}{R e}-q \text {. }
$$

Since $\mathcal{P}^{0} \equiv 0$, we set $\Theta^{q}(0)=0$, which implies $g(0, y)=$ $q(0)=0$. We impose $g(t, 0)=g(t, 1)=0$ so no velocity control effort is needed to steer the trajectory. Given these data, choosing $q$ completely determines $g$ from (10) and consequently $\Theta^{q}(t)$, so $q(t)$ parameterizes $\Theta^{q}(t)$.

In particular, choosing $q(t)$ as

$$
q(t)=\frac{8}{R e}\left(1-\mathrm{e}^{-c t}\right)
$$

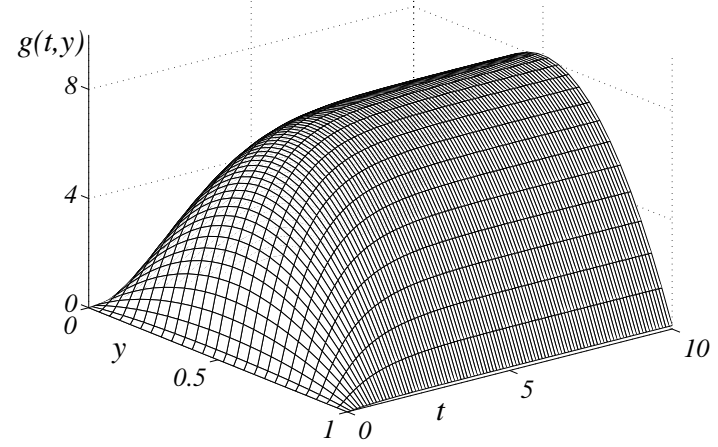

Fig. 2. Evolution of $g(t, y)$ for $c=1, R e=1$.

for $c>0$, then $q(0)=0$ and $\lim _{t \rightarrow \infty} q(t)=-8 / R e$.

Introducing (11) in (10), we can solve for $g$ analytically. Supposing $c \neq \pi^{2}(2 m+1)^{2} /$ Re for $m \in \mathbb{Z}, g$ is then

$$
\begin{aligned}
g= & 16 \sum_{m=0}^{m=\infty} \frac{\sin ((2 m+1) \pi y)}{(2 m+1)^{3} \pi^{3}} \\
& \times\left[1-\mathrm{e}^{-\frac{\pi^{2}(2 m+1)^{2}}{R e} t}-\frac{\mathrm{e}^{-c t}-\mathrm{e}^{-\frac{\pi^{2}(2 m+1)^{2}}{R e} t}}{1-\frac{c R e}{\pi^{2}(2 m+1)^{2}}}\right]
\end{aligned}
$$

As time grows, $g$ goes exponentially to its steady state

$$
\lim _{t \rightarrow \infty} g(t, y)=16 \sum_{m=0}^{m=\infty} \frac{\sin ((2 m+1) \pi y)}{(2 m+1)^{3} \pi^{3}}=4 y(1-y) .
$$

It can be proved as well ${ }^{1}$ that $g(t, y)$ is analytic on its domain of definition and verifies

$$
\begin{aligned}
|g(t, y)| & \leq 1 \\
\left|g_{y}(t, y)\right| & \leq 4
\end{aligned}
$$

In Figure 2 we represent $g$, computed numerically from (10), for $c=1, R e=1$.

Hence $\Theta^{q}(t)$ solves trajectory generation problem, since it verifies (1)-(5), $\Theta^{q}(0)=\mathcal{P}^{0}$ and $\lim _{t \rightarrow \infty} \Theta^{q}(t)=\mathcal{P}^{1}$. It follows that $\Theta^{q}(t)$ connects the chosen Poiseuille profiles ${ }^{2}$.

Using (9), we define the error variables as

$$
\begin{aligned}
(\tilde{u}, \tilde{v}, \tilde{p}) & =(u, v, p)-\Theta^{q}(t) \\
& =(u-g(t, y), v, p-x q(t)) .
\end{aligned}
$$

The error variables verify the following equations,

$$
\begin{aligned}
\tilde{u}_{t}= & \frac{\triangle \tilde{u}}{R e}-\tilde{p}_{x}-\tilde{u} \tilde{u}_{x}-\tilde{v} \tilde{u}_{y}-g(t, y) \tilde{u}_{x} \\
& -g_{y}(t, y) \tilde{v}, \\
\tilde{v}_{t}= & \frac{\triangle \tilde{v}}{R e}-\tilde{p}_{y}-\tilde{u} \tilde{v}_{x}-\tilde{v} \tilde{v}_{y}-g(t, y) \tilde{v}_{x},
\end{aligned}
$$

and the same boundary conditions and divergence-free condition as before. Our new objetive is to stabilize the equilibrium

\footnotetext{
${ }^{1}$ Using the maximum principle and other heat equation properties.

${ }^{2}$ Reaching $\mathcal{P}^{1}$ only after an infinitely long time, however by construction through rapidly decaying exponentials, $\Theta^{q}$ closely approaches $\mathcal{P}^{1}$ after a short time, as shown in Fig. 2. In this sense, we consider $\Theta^{q}$ a fast trajectory.
} 
at the origin in (17)-(18) by means of $U$ and $V$. This would imply that the trajectory $\Theta^{q}$ is stabilized.

Linearizing (17)-(18) around $\Theta^{q}$, and dropping tildes, we obtain the unsteady Stokes equations

$$
\begin{aligned}
u_{t} & =\frac{\triangle u}{R e}-p_{x}-g(t, y) u_{x}-g_{y}(t, y) v \\
v_{t} & =\frac{\triangle v}{R e}-p_{y}-g(t, y) v_{x}
\end{aligned}
$$

with boundary conditions

$$
\begin{aligned}
u(t, x, 0) & =v(t, x, 0)=0, \\
u(t, x, 1) & =U(t, x), \\
v(t, x, 1) & =V(t, x) .
\end{aligned}
$$

We aim to stabilize the origin of (19)-(20), hence achieving local stabilization $^{3}$ for the original nonlinear system.

\section{MAIN RESULTS}

Consider the following control laws.

The controller $V(t, x)$ is a dynamic controller, found as the unique solution of the following forced parabolic equation

$$
\begin{aligned}
V_{t}= & \frac{V_{x x}}{R e}-\sum_{0<|n|<M} \int_{-h}^{h} \mathrm{e}^{i \gamma_{n}(\xi-x)} \\
& \times\left[2 i \int_{0}^{1} g_{y}(t, \eta) \cosh \left(\gamma_{n}(1-\eta)\right) V(\tau, \xi, \eta) d \eta\right. \\
& \left.-i \frac{u_{y}(t, \xi, 0)-u_{y}(t, \xi, 1)}{R e}\right] d \xi
\end{aligned}
$$

initialized at zero, whereas the control law $U$ is given by

$$
U=\sum_{0<|n|<M} \int_{-h}^{h} \int_{0}^{1} \mathrm{e}^{i \gamma_{n}(\xi-x)} K_{n}(t, 1, \eta) u(t, \xi, \eta) d \eta d \xi
$$

where $M=\frac{2 h \sqrt{R e}}{\pi}$ and $\gamma_{n}=\pi n / h . K_{n}$ in (25) is the solution, for each $n$, of the following (well-posed)kernel equation

$$
\begin{aligned}
K_{n t}= & \frac{1}{R e}\left(K_{n y y}-K_{n \eta \eta}\right)-\lambda_{n}(t, \eta) K_{n}+f(y, \eta) \\
& -\int_{\eta}^{y} f(\xi, \eta) K_{n}(t, y, \xi) d \xi
\end{aligned}
$$

a linear partial integro-differential equation in the region $\Gamma=$ $(t, y, \eta) \in(0, \infty) \times \mathcal{T}$, where $\mathcal{T}=\left\{(y, \eta) \in \mathbb{R}^{2}: 0 \leq \eta \leq\right.$ $y \leq 1\}$, with boundary conditions:

$$
\begin{aligned}
K_{n}(t, y, y) & =-\operatorname{Re}\left(\lambda(y) \frac{y}{2}+\mu_{n}(0)\right) \\
K_{n}(t, y, 0) & =\operatorname{Re}\left[\int_{0}^{y} \mu_{n}(\sigma) K_{n}(t, y, \sigma) d \sigma-\mu_{n}(y)\right],(28)
\end{aligned}
$$

\footnotetext{
${ }^{3}$ Local stabilization suffices, since we assume the initial data are zero, i.e. the velocity field starts at the origin itself.
}

and where the functions that appear in (26)-(28) are

$$
\begin{aligned}
\lambda_{n}(t, y)= & i \gamma_{n} g(t, y) \\
f_{n}(t, y, \eta)= & -i \gamma_{n}\left[g_{y}(t, y)+2 \gamma_{n} \int_{\eta}^{y} g_{y}(t, \sigma)\right. \\
& \left.\times \sinh \left(\gamma_{n}(y-\sigma)\right) d \sigma\right] \\
\mu_{n}(y)= & \frac{\gamma_{n}}{R e} \frac{\left.\cosh \left(\gamma_{n}(1-y)\right)-\cosh \left(\gamma_{n} y\right)\right)}{\sinh \gamma_{n}}
\end{aligned}
$$

We state now our results.

Theorem 4.1: For any Reynolds number, the equilibrium $u \equiv v \equiv 0$ of Stokes system (19)-(23) with control laws (24)-(25) is exponentially stable in the $L^{2}$ norm, i.e., if $\mathbf{w}=$ $(u, v)$, there exist numbers $C_{1}(R e), C_{2}(R e)>0$ such that for $t \geq 0$,

$$
\|\mathbf{w}(t)\| \leq C_{1} \mathrm{e}^{-C_{2} t}\|\mathbf{w}(0)\| \text {. }
$$

The result above is valid for any initial condition. If we consider the nonlinear terms, local stability follows.

Theorem 4.2: For any Reynolds number, the equilibrium $u \equiv v \equiv 0$ of the Navier-Stokes system (17)-(18) with boundary conditions (21)-(23) and control laws (24)-(25) is locally exponentially stable in the $L^{2}$ norm, i.e., if $\mathbf{w}=$ $(u, v)$, there exist numbers $\epsilon(R e), C_{1}(R e), C_{2}(R e)>0$ such that if $\|\mathbf{w}(0)\|<\epsilon$, and for $t \geq 0$,

$$
\|\mathbf{w}(t)\| \leq C_{1} \mathrm{e}^{-C_{2} t}\|\mathbf{w}(0)\| .
$$

From Section III and Theorem 4.2, the final result follows.

Theorem 4.3: For any Reynolds number, $\Theta^{q}(t)$ defined by (9)-(12) is a solution of system (1)-(5), with imposed pressure gradient (11), and control laws (24)-(31) expressed in the error variables (16). Moreover, this solution is locally exponentially stable in the $L^{2}$ norm. In particular, if the state is initialized close enough to rest, it closely follows $\Theta^{q}(t)$ and approaches the steady equilibrium $\mathcal{P}^{1}$ exponentially fast.

Remark 1: Even though the controller (24)-(31) looks rather involved, it is not hard to implement. A finite set of linear PIDE equations has to be solved for computing the controllers, which can be done fast and efficiently [13].

Remark 2: This result can be extended in a number of ways. An output feedback design is possible applying a dual backstepping observer methodology [15], [18], only requiring boundary measurements of pressure and skin friction. A 3D channel flow, periodic in two directions, is also tractable, adding some refinements which include actuation of the spanwise velocity at the wall. Stability in the $H^{1}, H^{2}$ norms can be obtained as well. We skip the details due to page limit.

Remark 3: From (24) it follows that the mean of $V$ is zero, hence verifying the zero net flux condition.

In the next sections we introduce a mathematical framework and prove some of the results, skipping some proofs due to space restriction. Full details will be provided in a future publication. 


\section{MATHEMATICAL PRELIMINARIES}

\section{A. Periodic function spaces}

We assume that the velocity field $(u, v)$ and the pressure $p$ are periodic in $x$ with some period $2 h>0$ [16]. This requires for consistency that $U$ and $V$ are periodic with the same period; a property already verified by expressions (24)(25). $\Omega$ and its boundary are identified with

$$
\begin{aligned}
\Omega_{h} & =\{(x, y) \in \Omega:-h \leq x \leq h\}, \\
\partial \Omega_{h i} & =\left\{(x, y) \in \partial \Omega_{i}:-h \leq x \leq h\right\} .
\end{aligned}
$$

Let $L^{2}\left(\Omega_{h}\right)$ be the usual Lebesgue space of square-integrable functions, endowed with the scalar product

$$
(\phi, \psi)_{L^{2}\left(\Omega_{h}\right)}=\int_{-h}^{h} \int_{0}^{1} \phi(x, y) \psi(x, y) d y d x .
$$

Define then $L_{h}^{2}(\Omega)=L^{2}\left(\Omega_{h}\right)$, where now

$$
(\phi, \psi)_{L_{h}^{2}(\Omega)}=\left(\left.\phi\right|_{\Omega_{h}},\left.\psi\right|_{\Omega_{h}}\right)_{L^{2}\left(\Omega_{h}\right)} .
$$

\section{B. Fourier series expansion}

Given a function $\phi$ we define the sequence of its complex Fourier coefficients $\left(\phi_{n}(y)\right)_{n \in \mathbb{Z}}$ as

$$
\phi_{n}(y)=\frac{1}{2 h} \int_{-h}^{h} \phi(x, y) \mathrm{e}^{\frac{i n \pi}{h} x} d x, \quad n \in \mathbb{Z} .
$$

We will simply write $\phi_{n}$ in the sequel. It can be shown that if $\phi \in L^{2}\left(\Omega_{h}\right)$, then (38) is well defined and $\phi_{n}$ is in the (complex valued) $\ell^{2} \times L^{2}(0,1)$ space, i.e.,

$$
\sum_{n \in \mathbb{Z}} \int_{0}^{1}\left|\phi_{n}(y)\right|^{2} d y<\infty
$$

One can recover $\phi$ by writting its Fourier series,

$$
\phi(x, y)=\sum_{n \in \mathbb{Z}} \phi_{n}(t, y) \mathrm{e}^{-\frac{i n \pi}{h} x} .
$$

Equation (40) yields a $L^{2}\left(\Omega_{h}\right)$ function if $\phi_{n} \in \ell^{2} \times L^{2}(0,1)$.

One important result is Parseval's formula

$$
(\phi, \psi)_{L^{2}\left(\Omega_{h}\right)}=\left(\phi_{n}, \psi_{n}\right)_{\ell^{2} \times L^{2}(0,1)}
$$

where the $\ell^{2} \times L^{2}(0,1)$ scalar product is

$$
\left(\phi_{n}, \psi_{n}\right)_{\ell^{2} \times L^{2}(0,1)}=\sum_{n \in \mathbb{Z}} \int_{0}^{1} \phi_{n}(y) \bar{\psi}_{n}(y) d y,
$$

and where the bar denotes the complex conjugate.

In the sequel we frequently omit the subindexes when clear from the context.

Using (41), and given $\psi$ in $L^{2}\left(\Omega_{h}\right)$, we can compute its norm by computing its Fourier coefficients $\psi_{n}$. Then,

$$
\|\psi\|_{L^{2}\left(\Omega_{h}\right)}^{2}=\|\psi\|_{\ell^{2} \times L^{2}(0,1)}^{2}=\sum\left\|\psi_{n}\right\|_{L^{2}(0,1)}^{2},
$$

where

$$
\left\|\psi_{n}\right\|_{L^{2}(0,1)}^{2}=\int_{0}^{1}\left|\psi_{n}(y)\right|^{2} d y .
$$

\section{C. $H^{1}$ spaces}

We define the space $H_{h}^{1}(\Omega)$ as

$$
H_{h}^{1}(\Omega)=\left\{\left.f\right|_{\Omega_{h}} \in H^{1}\left(\Omega_{h}\right),\left.f\right|_{x=-h}=\left.f\right|_{x=h} \text { a.e. }\right\} \text {. }
$$

The $H^{1}$ norm is defined as

$$
\|\phi\|_{H_{h}^{1}(\Omega)}^{2}=\|\phi\|_{L_{h}^{2}(\Omega)}^{2}+\left\|\phi_{y}\right\|_{L_{h}^{2}(\Omega)}^{2}+\left\|\phi_{x}\right\|_{L_{h}^{2}(\Omega)}^{2},
$$

or in terms of the Fourier coefficients

$$
\begin{aligned}
\|\phi\|_{H_{h}^{1}(\Omega)}^{2}= & \sum\left[\left(1+4 \pi^{2} n^{2}\right)\left\|\phi_{n}\right\|_{L^{2}(0,1)}^{2}\right. \\
& \left.+\left\|\phi_{n y}\right\|_{L^{2}(0,1)}^{2}\right] .
\end{aligned}
$$

We state the following lemma, whose proof we skip.

Lemma 5.1: Suppose that $\phi \in H_{h}^{1}(\Omega)$ such that $\left.\phi\right|_{\partial \Omega_{0}} \equiv$ 0 , and $\psi \in L_{h}^{2}(\Omega)$. Then:

$$
\left(\phi^{2}, \psi^{2}\right)_{L_{h}^{2}(\Omega)} \leq\left\|\phi_{y}\right\|_{L_{h}^{2}(\Omega)}^{2}\|\psi\|_{L_{h}^{2}(\Omega)}^{2} .
$$

\section{Spaces for the velocity field}

Calling $\mathbf{w}=(u, v)$, we define

$$
\begin{aligned}
& H_{0 h}(\Omega)=\left\{\mathbf{w} \in\left[L_{h}^{2}(\Omega)\right]^{2}: \nabla \cdot \mathbf{w}=0,\left.\mathbf{w}\right|_{\partial \Omega_{0}}=0\right\} \\
& H_{0 h}^{1}(\Omega)=H_{0 h}(\Omega) \cap\left[H_{h}^{1}(\Omega)\right]^{2}
\end{aligned}
$$

endowed with the scalar product of, respectively, $\left[L_{h}^{2}(\Omega)\right]^{2}$ and $\left[H_{h}^{1}(\Omega)\right]^{2}$. See [16] for the precise meaning of divergence and trace in this space.

\section{E. Transformations of $L^{2}$ functions}

Our approach uses the backstepping method [13]. The method is based on finding a invertible mapping of the state variables into others with desired stability properties. We study the kind of transformations that appear in the method.

Definition 5.1: Given complex valued functions $f \in$ $L^{2}(0,1)$ and $K \in L^{\infty}(\mathcal{T})$, we define the transformed variable $g=(\mathrm{I}-K) f$, where the operator $K f$ is defined as

$$
K f=\int_{0}^{y} K(y, \eta) f(\eta) d \eta,
$$

i.e. a Volterra operator. We call $\mathrm{I}-K$ the direct transformation with kernel $\mathrm{K}$. If there exists a function $L \in L^{\infty}(\mathcal{T})$ such that $f=(\mathrm{I}+L) g$, then we say that the transformation is invertible, and we call $\mathrm{I}+L$ the inverse transformation, and $L$ the inverse kernel (or the inverse of $K$ ).

We state some important results [7].

Proposition 5.1: For $K \in L^{\infty}(\mathcal{T})$, the transformation I$K$ is always invertible. Moreover, $L$ is related to $K$ by

$$
\begin{aligned}
L(y, \eta) & =K(y, \eta)+\int_{\eta}^{y} K(y, \sigma) L(\sigma, \eta) d \sigma \\
& =K(y, \eta)+\int_{\eta}^{y} L(y, \sigma) K(\sigma, \eta) d \sigma .
\end{aligned}
$$

Proposition 5.2: If $f \in L^{2}(0,1)$ then $g=(\mathrm{I}-K) f$ is in $L^{2}(0,1)$. Similarly, if $g \in L^{2}(0,1)$ then $f=(\mathrm{I}+L) g$ is in $L^{2}(0,1)$. Moreover,

$$
\begin{aligned}
\|g\|_{L^{2}(0,1)}^{2} & \leq\left(1+\|K\|_{\infty}\right)^{2}\|f\|_{L^{2}(0,1)}^{2}, \\
\|f\|_{L^{2}(0,1)}^{2} & \leq\left(1+\|L\|_{\infty}\right)^{2}\|g\|_{L^{2}(0,1)}^{2} .
\end{aligned}
$$


Proposition 5.2 allows to define an $L^{2}$ equivalent norm,

$$
\|f\|_{K L^{2}(0,1)}^{2}=\|(\mathrm{I}-K) f\|_{L^{2}(0,1)}^{2}=\|g\|_{L^{2}(0,1)}^{2} .
$$

For $\mathcal{C}^{1}(\mathcal{T})$ kernels $K$ and $L$, one has an equivalent version of Proposition 5.1 and Proposition 5.2, allowing to define a $K H^{1}(0,1)$ norm, which is equivalent to the $H^{1}(0,1)$ norm.

\section{F. Transformations of the velocity field}

Definition 5.2: Consider $A=\left\{a_{1}, \ldots, a_{j}\right\} \subset \mathbb{Z}$ and $\mathcal{K}=$ $\left(K_{n}(y, \eta)\right)_{n \in A}$ a family of $L^{\infty}(\mathcal{T})$ kernels. Then, for $\mathbf{w}=$ $(u, v) \in H_{0 h}(\Omega)$, one defines the transformed variable $\omega=$ $(\alpha, \beta)=(\mathrm{I}-\mathcal{K}) \mathbf{w}$, through its Fourier components,

$$
\omega_{n}=\left\{\begin{array}{c}
\left(\left(\mathrm{I}-K_{n}\right) u_{n}, 0\right) n \in A \\
\mathbf{w}_{n}, \text { otherwise. }
\end{array}\right.
$$

The inverse transformation, $\mathbf{w}=(I+\mathcal{L}) \omega$, is defined as

$$
\mathbf{w}=\left\{\begin{array}{c}
\left(\left(\mathrm{I}+L_{n}\right) \alpha_{n}, \hat{L}_{n} \alpha_{n}\right) n \in A, \\
\omega_{n}, \text { otherwise, }
\end{array}\right.
$$

where the new operator $\hat{L}_{n}$ is defined as:

$$
\hat{L}_{n} f=-\pi i \frac{n}{h} \int_{0}^{y}\left(f(\eta)+\int_{0}^{\eta} L(\eta, \sigma) f(\sigma) d \sigma\right) d \eta .
$$

Using the divergence-free condition in Fourier space, $\pi i \frac{n}{h} u_{n}+v_{n y}=0$, and the boundary condition $v_{n}(0)=0$, it is straightforward to show that the inverse is correctly defined.

Even though $v_{n}$ is apparently lost, it can be recovered since the transformation is invertible. Using a similar argument as in Proposition 5.2,

$$
\begin{aligned}
\|\omega\|_{H_{0 h}(\Omega)}^{2} & \leq\left(1+\|\mathcal{K}\|_{\infty}\right)^{2}\|\mathbf{w}\|_{H_{0 h}(\Omega)}^{2}, \\
\|\mathbf{w}\|_{H_{0 h}(\Omega)}^{2} & \leq\left(1+N^{2}\right)\left(1+\|\mathcal{L}\|_{\infty}\right)^{2}\|\omega\|_{H_{0 h}(\Omega)}^{2},
\end{aligned}
$$

where $N=\max _{n \in A}\left\{\pi \frac{n}{h}\right\}$, and

$$
\begin{aligned}
\|\mathcal{K}\|_{\infty} & =\max _{n \in A}\left\{\left\|K_{n}\right\|_{\infty}\right\}, \\
\|\mathcal{L}\|_{\infty} & =\max _{n \in A}\left\{\left\|L_{n}\right\|_{\infty}\right\} .
\end{aligned}
$$

This allows the definition of an $H_{0 h}(\Omega)$ equivalent norm, as in (55), that we call $\mathcal{K} H_{0 h}(\Omega)$.

\section{PRoOF OF THEOREM 4.1}

Equations (19)-(20) in Fourier space are

$$
\begin{aligned}
& u_{n t}=\frac{\triangle_{n} u_{n}}{R e}-i \gamma_{n}\left(p_{n}+g(t, y) u_{n}\right)-g_{y}(t, y) v_{n} \\
& v_{n t}=\frac{\triangle_{n} v_{n}}{R e}-p_{n y}-i \gamma_{n} g(t, y) v_{n}
\end{aligned}
$$

where $\triangle_{n}=\partial_{y y}-\gamma_{n}^{2}$. The boundary conditions are

$$
\begin{aligned}
& u_{n}(t, 0)=v_{n}(t, 0)=0, \\
& u_{n}(t, 1)=U_{n}(t), \\
& v_{n}(t, 1)=V_{n}(t),
\end{aligned}
$$

and the divergence-free condition becomes

$$
i \gamma_{n} u_{n}+v_{n y}=0 \text {. }
$$

From (63)-(64) an equation for the pressure can be derived,

$$
p_{n y y}-\gamma_{n}^{2} p_{n}=-2 i \gamma_{n} g_{y}(t, y) v_{n}
$$

with boundary conditions obtained from evaluating (64) at the boundaries and using (66)-(67),

$$
\begin{aligned}
& p_{n y}(0, t)=-i \gamma_{n} \frac{u_{n y}(0, t)}{R e} \\
& p_{n y}(1, t)=-i \gamma_{n} \frac{u_{n y}(1, t)}{R e}-\dot{V}_{n}-\gamma_{n}^{2} \frac{V_{n}}{R e} .
\end{aligned}
$$

Equations for different $n$ 's are uncoupled, allowing separate consideration for each mode. Most modes, which we refer to as uncontrolled, are naturally stable and thus left without control. A finite set of modes, called controlled, are unstable and require control.

\section{A. Uncontrolled modes}

1) $n=0$ (mean velocity field): From (68), $v_{0} \equiv 0 . u_{0}$ verifies

$$
u_{0 t}=\frac{u_{0 y y}}{R e}
$$

with $u_{0}(0)=u_{0}(1)=0$. The following estimate holds:

$$
\frac{d}{d t}\left\|u_{0}(t)\right\|_{L^{2}(0,1)}^{2} \leq \mathrm{e}^{-\frac{2}{R e} t}\left\|u_{0}(0)\right\|_{L^{2}(0,1)}^{2} .
$$

2) Modes for large $|n|$ : If $\mathbf{w}_{n}=\left(u_{n}, v_{n}\right)$, then, considering no control $\left(V_{n}=U_{n}=0\right)$ :

$$
\begin{aligned}
\frac{d}{d t}\left\|\mathbf{w}_{n}\right\|^{2}= & -2 \frac{\left\|\mathbf{w}_{n y}\right\|^{2}}{R e}-2 \gamma_{n}^{2} \frac{\left\|\mathbf{w}_{n}\right\|^{2}}{R e}-\left(g_{y} u_{n}, v_{n}\right) \\
& -\left(g_{y} v_{n}, u_{n}\right)-\left(u_{n}, i \gamma_{n} p_{n}\right)-\left(i \gamma_{n} p_{n}, u_{n}\right) \\
& -\left(v_{n}, p_{n y}\right)-\left(p_{n y}, v_{n}\right) .
\end{aligned}
$$

Consider the pressure terms like those in the last two lines of (74). Using (68) and integration by parts,

$$
-\left(u_{n}, i \gamma_{n} p_{n}\right)=-\left(v_{n y}, p_{n}\right)=\left(v_{n}, p_{n y}\right)
$$

Then, using Young's inequality with the remaining terms,

$$
\begin{aligned}
\frac{d}{d t}\left\|\mathbf{w}_{n}\right\|^{2} \leq & -2 \frac{\left\|\mathbf{w}_{n y}\right\|^{2}}{R e}-2 \gamma_{n}^{2} \frac{\left\|\mathbf{w}_{n}\right\|^{2}}{R e} \\
& +\left\|g_{y}\right\|_{L_{\infty}}\left\|\mathbf{w}_{n}\right\|^{2} .
\end{aligned}
$$

Since $\left|g_{y}(t, y)\right| \leq 4$, choosing $\left|\gamma_{n}\right| \geq \sqrt{2 R e}$, i.e.,

$$
|n| \geq M=\frac{2 h \sqrt{R e}}{\pi}
$$

yields

$$
\frac{d}{d t}\left\|\mathbf{w}_{n}\right\|^{2} \leq-2 \frac{\left\|\mathbf{w}_{n y}\right\|^{2}}{R e}-\gamma_{n}^{2} \frac{\left\|\mathbf{w}_{n}\right\|^{2}}{R e} \leq-2 \frac{\left\|\mathbf{w}_{n}\right\|^{2}}{R e}
$$

by Poincare's inequality, therefore achieving $L^{2}$ exponential stability for large modes $(|n| \geq M)$.

\section{B. Controlled modes. Construction of control laws}

The modes $0<|n|<M$ are open-loop unstable and must be controlled. We design the control in several steps. 
1) Pressure shaping: Solving (69)-(71),

$$
\begin{aligned}
p_{n}= & -2 i \int_{0}^{y} g_{y}(t, \eta) \sinh \left(\gamma_{n}(y-\eta)\right) v_{n}(t, \eta) d \eta \\
& +2 i \frac{\cosh \left(\gamma_{n} y\right)}{\sinh \gamma_{n}} \int_{0}^{1} g_{y}(t, \eta) \cosh \left(\gamma_{n}(1-\eta)\right) \\
& \times v_{n}(t, \eta) d \eta+i \frac{\cosh \left(\gamma_{n}(1-y)\right)}{\sinh \gamma_{n}} \frac{u_{n y}(0, t)}{R e} \\
& -\frac{\left.\cosh \left(\gamma_{n} y\right)\right)}{\sinh \gamma_{n}}\left(i \frac{u_{n y}(1, t)}{R e}+\frac{\dot{V}_{n}}{\gamma_{n}}+\gamma_{n} \frac{V_{n}}{R e}\right) .
\end{aligned}
$$

We set $V_{n}$ to enforce in (79) a strict-feedback structure [10] in $y$. This property, required by backstepping [13], [14], is a sort of "spatial causality". We choose $V_{n}$ as

$$
\begin{aligned}
\frac{\dot{V}_{n}}{\gamma_{n}}= & -\gamma_{n} \frac{V_{n}}{R e}-i \frac{u_{n y}(0, t)-u_{n y}(1, t)}{R e} \\
& -2 i \int_{0}^{1} g_{y}(t, \eta) \cosh \left(\gamma_{n}(1-\eta)\right) v_{n}(t, \eta) d \eta .
\end{aligned}
$$

2) Control of velocity field: By (68), $v_{n}$ can be computed from $u_{n}$. Then, only $u_{n}$ is needed. Using (68) to eliminate $v_{n}$ and introducing (80) and (79) into (63), yields

$$
\begin{aligned}
u_{n t}= & \frac{\triangle_{n} u_{n}}{R e}+\lambda_{n}(t, y) u_{n}+\int_{0}^{y} f_{n}(t, y, \eta) u_{n}(t, \eta) d \eta \\
& +\mu_{n}(y) u_{n y}(0, t),
\end{aligned}
$$

with $\lambda_{n}, f_{n}$ and $\mu_{n}$ as in (29)-(31), and boundary conditions

$$
\begin{aligned}
& u_{n}(t, 0)=0, \\
& u_{n}(t, 1)=U_{n}(t) .
\end{aligned}
$$

Following [14] we map $u_{n}$, for each mode $0<|n|<M$, into the family of heat equations:

$$
\begin{aligned}
\alpha_{n t} & =\frac{1}{R e}\left(-\gamma_{n}^{2} \alpha_{n}+\alpha_{n y y}\right) \\
\alpha_{n}(k, 0) & =\alpha_{n}(k, 1)=0,
\end{aligned}
$$

where

$$
\begin{aligned}
& \alpha_{n}=\left(I-K_{n}\right) u_{n} \\
& u_{n}=\left(I+L_{n}\right) \alpha_{n},
\end{aligned}
$$

are respectively the direct and inverse transformations. The kernel $K_{n}$ is found to verify equations (26)-(28), which can be proved well-posed, and $L_{n}$ can be derived from $K_{n}$.

The control law is, from (86), (85) and (83)

$$
U_{n}=\int_{0}^{1} K_{n}(t, 1, \eta) u_{n}(t, k, \eta) d \eta
$$

Stability follows from (84)-(85) and (86)-(87). We obtain

$$
\frac{d}{d t}\left\|u_{n}\right\|_{K_{n} L^{2}(0,1)}^{2} \leq \mathrm{e}^{-\frac{2}{R e} t}\left\|u_{n}(0)\right\|_{K_{n} L^{2}(0,1)}^{2} .
$$

\section{Stability for the whole system}

If we call $A=\{n \in \mathbb{Z}: 0<|n|<M\}$, and $\mathcal{K}=K_{n}(t, y, \eta)_{n \in A}$, and apply the control laws (88)-(80) in physical space, which yield (25)-(24), then it follows that

$$
\begin{aligned}
\|\mathbf{w}\|_{\mathcal{K}_{H_{0 h}(\Omega)}}^{2}= & \sum_{n \notin A}\left\|\mathbf{w}_{n}\right\|_{L^{2}(0,1)^{2}}^{2}+\sum_{n \in A}\left\|u_{n}\right\|_{K_{n} L^{2}(0,1)}^{2} \\
\leq & \mathrm{e}^{-\frac{2}{R e} t}\left[\sum_{n \notin A}\left\|\mathbf{w}_{n}(0)\right\|_{L^{2}(0,1)^{2}}^{2}\right. \\
& \left.+\sum_{n \in A}\left\|u_{n}(0)\right\|_{K_{n} L^{2}(0,1)}^{2}\right] \\
\leq & \mathrm{e}^{-\frac{2}{R e} t}\|\mathbf{w}(0)\|_{\mathcal{K}_{H_{0 h}(\Omega)}}^{2},
\end{aligned}
$$

and by norm equivalency, this proves Theorem 4.1.

\section{ACKNOWLEDGEMENTS}

We acknowledge the support of a EU Marie Curie Fellowship, in the framework of the CTS, contract number HPMTCT-2001-00278. We thank T. Bewley, M. Krstic and A. Smyshlyaev for valuable suggestions for helpful discussions.

\section{REFERENCES}

[1] O. M. Aamo and M. Krstic, Flow Control by Feedback: Stabilization and Mixing, Springer, 2002.

[2] A. Balogh, W.-J. Liu and M. Krstic, "Stability enhancement by boundary control in 2D channel flow," IEEE Transactions on Automatic Control, vol. 46, pp. 1696-1711, 2001.

[3] G. K. Batchelor, An Introduction to Fluid Mechanics, Cambridge University Press, London, 1967.

[4] J.-M. Coron and E. Trélat, "Global steady-state controllability of 1D semilinear heat equations," SIAM J. Contr. and Opt., vol. 43, pp. 549$569,2004$.

[5] J.-M. Coron and E. Trelat, "Global steady-state stabilization and controllability of 1D semilinear wave equations," to appear, Commun. Contemp. Math., 2006.

[6] J.-M. Coron, " Local controllability of a 1D tank containing a fluid modeled by the shallow water equations," ESAIM: Contr. Optim. Calc. Variat., vol. 8, pp. 513-554, 2002.

[7] H. Hochstadt, Integral Equations, Wiley, 1973.

[8] M. Hogberg, T. R. Bewley and D. S. Henningson, "Linear feedback control and estimation of transition in plane channel flow," Journal of Fluid Mechanics, vol. 481, pp. 149-175, 2003.

[9] C. H. von Kerczek, "The instability of oscillatory plane Poiseuille flow," Journal of Fluid Mechanics, vol. 116, pp. 91-114, 1982

[10] M. Krstic, I. Kanellakopoulos and P. V. Kokotovic, Nonlinear and Adaptive Control Design, Wiley, 1995.

[11] P. J. Schmid and D. S. Henningson. Stability and Transition in Shear Flows, Springer, 2001.

[12] M. Schmidt, E. Trélat, "Controllability of Couette flows", Comm. Pure Applied Analysis 5, 1 (2006), 201-211.

[13] A. Smyshlyaev and M. Krstic, "Closed form boundary state feedbacks for a class of partial integro-differential equations," IEEE Transactions on Automatic Control, vol. 49, pp. 2185-2202, 2004.

[14] A. Smyshlyaev and M. Krstic, "On control design for PDEs with space-dependent diffusivity or time-dependent reactivity," Automatica, vol. 41, pp. 1601-1608, 2005 .

[15] A. Smyshlyaev and M. Krstic, "Backstepping observers for parabolic PDEs," Systems and Control Letters, vol. 54, pp. 1953-1971, 2005.

[16] R. Temam, Navier-Stokes Equations, Theory and Numerical Analysis, North-Holland Publishing Co., Amsterdam, 1984.

[17] R. Vázquez and M. Krstic, "A closed-form feedback controller for stabilization of linearized Navier-Stokes equations: the 2D Poisseuille flow," Procs. of the 44th CDC, Sevilla, 2005.

[18] R. Vázquez and M. Krstic, "A closed-form observer for the channel flow Navier-Stokes system," Procs. of the 44th CDC, Sevilla, 2005. 